\title{
LEVEL SETS OF SCALAR WEYL INVARIANTS AND COHOMOGENEITY
}

\author{
SERGIO CONSOLE AND CARLOS OLMOS
}

To the memory of Aristide Sanini

\begin{abstract}
We prove that the cohomogeneity of a Riemannian manifold coincides locally with the codimension of the foliation by regular level sets of the scalar Weyl invariants.
\end{abstract}

\section{INTRODUCTION}

A tensorial Weyl invariant is a tensor $T$ on a Riemannian manifold $M$ which can be naturally defined in terms of the metric, the curvature and its covariant derivatives. For example, the scalar curvature is a real-valued tensorial Weyl invariant of type $(0,0)$. If a tensorial Weyl invariant is in fact a function, it is called a scalar Weyl invariant. Tensorial Weyl invariants are natural tensors, i.e., they are invariant under local isometries with any Riemannian manifold [2].

If some tensorial Weyl invariants on a Riemannian manifold $M$ are "independent of the point", then there are strong consequences on the geometry of $M$. For instance, a classical result of É. Cartan says that, if the covariant derivative of the curvature tensor vanishes (hence the curvature is invariant by parallel transport), $M$ is locally symmetric. More generally, a theorem of Singer [7] asserts that $M$ is locally homogeneous if the algebraic type of all covariant derivatives $\nabla^{\ell} R_{p}$ at $p \in M$ of the curvature tensor (up to some index $k$ ) is independent of $p$ (i.e., for any $q \in M$ there is a linear isometry $f$ of the tangent spaces at $p$ and $q$ such that $\left.f^{*} \nabla^{\ell} R_{q}=\nabla^{\ell} R_{p}\right)$. Using Singer's result, Prüfer, Tricerri and Vanhecke showed that if $M$ has globally constant scalar Weyl invariants, then $M$ is locally homogeneous [6].

In this paper, we consider the general situation where the scalar Weyl invariants are not constant. What one can do is to look at their regular level sets.

Example 1. Let $M \subset \mathbb{R}^{n+1}$ be a hypersurface of revolution, i.e., the group $G_{\ell} \cong S O(n)$ of rotations around a line $\ell$ in $\mathbb{R}^{n+1}$ leaves $M$ invariant. If $M$ is generic

Received by the editors July 28, 2005.

2000 Mathematics Subject Classification. Primary 53C30; Secondary 53C21.

Key words and phrases. Homogeneous Riemannian manifolds, Weyl invariants, natural tensors, cohomogeneity.

The first author was partially supported by GNSAGA of INdAM and MIUR (Italy).

The second author was supported by Universidad Nacional de Córdoba and CONICET, and partially supported by Antorchas, ANCyT, Secyt-UNC, and CIEM.

(C) 2007 American Mathematical Society Reverts to public domain 28 years from publication 
(e.g., not homogeneous, itself), $G_{\ell}$ is the connected component of the identity of the full isometry group. The principal $G_{\ell}$-orbits are totally umbilical hyperspheres. A hypersurface of revolution provides a basic example of a cohomogeneity one manifold (and conversely, under some assumptions any cohomogeneity one hypersurface is of revolution; cf. for instance [5]). Observe that, by equivariance, the scalar Weyl invariants are constant on the orbits. Thus the principal orbits are contained in the regular level sets of the scalar Weyl invariants. Actually, for dimensional reasons, the principal orbits are locally the regular level sets of the scalar Weyl invariants.

In general, on a cohomogeneity $k G$-manifold $M(G \subseteq I(M))$ the scalar Weyl invariants are constant on the $G$-orbits. Thus the principal $G$-orbits are contained in the regular level sets of the scalar Weyl invariants. We prove that, locally, we have in fact equality.

Theorem 1. The cohomogeneity of a Riemannian manifold $M$ (with respect to the full isometry group) coincides locally with the codimension of the foliation by regular level sets of the scalar Weyl invariants.

The proof requires some properties of Riemannian submersions, which will be applied to the projection $\pi$ of $M$ onto the quotient manifold $N$ obtained by identifying points lying in the same level set (endowed with a Riemannian metric so that $\pi$ becomes a Riemannian submersion). Roughly speaking, we have to deal with the holonomy of such a submersion.

In Section 2 we start with recalling some properties of Riemannian submersions. Then, we consider parallel transport on the fibers and we introduce a notion of covariant derivative and curvature applied to vector and tensor fields on the fibers. We state a condition for the vertical foliation of a Riemannian submersion to be locally given by orbits of the isometry group of the ambient space (Theorem 2). This condition involves the behavior of Killing vector fields on the fibers. Namely, suppose at any point $q$ on the base space there exists a family $\mathcal{K}(q)$ of Killing vector fields defined on the fiber at $q$, with the property that the Killing fields are invariant by parallel transport across the fibers and any Killing in $\mathcal{K}(q)$ commutes with the curvature vector fields on the fibers. Then Theorem 2 states that any Killing in $\mathcal{K}(q)$ extends to a vertical Killing vector field on the whole space. In particular, if the pseudogroup of isometries $K(q)$ associated with $\mathcal{K}(q)$ is locally transitive, the vertical foliation is locally homogeneous. This will be an important tool for the proof of Theorem 1.

In Section 3 we study level sets of scalar Weyl invariants. As a start, using the above mentioned theorem of Singer, we show that any level set is locally (intrinsically) homogeneous (Corollary 1). We introduce certain finite dimensional subbundles of the bundle $\Gamma^{m, k}$ of tensor fields of type $(m, k)$ on fibers, which turn out to be parallel. One of these bundles, denoted by $E^{(1,0)}$, is in fact strictly related to the holonomy subbundle of $p$ (that is, the set of points that can be locally reached by horizontal curves starting at $p$; cf. [8] and Remark 6).

Let $\mathcal{K}(q)$ be the family of (locally defined) Killing of the fiber $\pi^{-1}(\{q\})$ whose associated flow preserve any of the tensor fields of the above bundles. We show that this family $\mathcal{K}(q)$ fulfills the conditions of Theorem 2 . Thus, the foliation by the regular level set of scalar Weyl invariants is homogeneous. This yields the proof of Theorem 1. 


\section{Preliminaries AND BASIC FACtS}

2.1. Riemannian submersions. We recall some basic definitions and properties of Riemannian submersions.

Let $\pi: M \rightarrow N$ be a Riemannian submersion. This means that $\pi$ is onto and $d \pi_{\mid * p}$ is surjective, for all $p \in M$. Moreover, for all $p \in M, d \pi: \mathcal{H}_{p} \rightarrow T_{\pi(p)} N$ is a linear isometry, where the so-called horizontal distribution is $\mathcal{H}:=\nu^{\perp}$ and $\nu$ is the vertical distribution (i.e. $\nu=\operatorname{ker} d \pi$ or equivalently $\nu_{p}$ is the tangent space at $p$ to the fiber $\left.\pi^{-1}(\{\pi(p)\})\right)$.

A vector $v \in T_{p} M$ is called horizontal if $v \in \mathcal{H}_{p}$ and vertical if $v \in \nu_{p}$. A vector field $X$ of $M$ is called:

- vertical if $X$ lies in $\nu$;

- horizontal if $X$ lies in $\mathcal{H}$;

- projectable if $X$ is $\pi$-related to some field $Y$ on $N$;

- basic if $X$ is horizontal and projectable.

Given a (vector) field $Y$ on $N$ there is a unique field $\tilde{Y}$ of $M$ which is basic and $\pi$-related to $Y$, the horizontal lift of $X$. A piecewise $\mathcal{C}^{1}$ curve $c:[a, b] \rightarrow M$ is said to be horizontal if $c^{\prime}(t)$ is a horizontal vector, for all $t \in[a, b]$. If $\beta:[a, b] \rightarrow N$ is a piecewise $\mathcal{C}^{1}$ curve, then, given any $q \in \pi^{-1}(\{\beta(a)\})$, there is a unique horizontal curve $\tilde{\beta}^{q}:[a, a+\varepsilon] \rightarrow M$ such that $\tilde{\beta}^{q}(a)=q$ and $\pi \circ \tilde{\beta}^{q}=\beta_{\mid[a, a+\varepsilon]}$ ( $\varepsilon$ depends on $q$ ). The curve $\tilde{\beta}^{q}$ is the so-called (local) horizontal lift of $\beta$ through $q$. If $M$ is complete, then $\tilde{\beta}$ is defined in $[a, b]$. So, one has the map

$$
\tau_{\beta}: \pi^{-1}(\{\beta(a)\}) \rightarrow \pi^{-1}(\{\beta(b)\})
$$

defined by $\tau_{\beta}(x)=\tilde{\beta}^{x}(b)$. The map $\tau_{\beta}$ is the parallel transport along $\beta$ and does not depend on the parametrization of the curve. Moreover, $\tau_{\beta}$ is a diffeomorphism with inverse $\tau_{\tilde{\beta}}$, where $\tilde{\beta}$ is the curve $\beta$ going in the backwards direction. Any horizontal lift of a geodesic of $N$ is always a geodesic of $M$. So, if $M$ is complete, then $N$ is complete (and the fibers $\pi^{-1}(\{q\})$ are also complete since they are closed subsets of $M)$.

If $X_{1}, X_{2}$ are projectable fields of $M$, then $\left[X_{1}, X_{2}\right]$ is also projectable, since it projects down to the bracket of the projections of $X_{1}$ and $X_{2}$. If $X_{1}, X_{2}$ are basic, then $\left[X_{1}, X_{2}\right]$ is a projectable field but not in general basic. Otherwise, the distribution $\mathcal{H}$ would be involutive or equivalently integrable. How far is $\mathcal{H}$ from being involutive is measured by the tensor $O$ of $M$, defined by

$$
O_{Z_{1}, Z_{2}}=\left[Z_{1}^{\mathcal{H}}, Z_{2}^{\mathcal{H}}\right]^{\nu}
$$

i.e., the vertical part of the bracket of the horizontal components of the fields. It turns out that $O$ is a tensor field. The tensor $\frac{1}{2} O$ is merely the so-called O'Neill tensor $A$, composed with the horizontal projection (see [4]). A pair of fields $X_{1}, X_{2}$ of $N$ define the vertical field $R_{X_{1}, X_{2}}^{\pi}$ of $M$ given by

$$
R_{X_{1}, X_{2}}^{\pi}:=O_{\tilde{X}_{1}, \tilde{X}_{2}}
$$

where $\tilde{X}_{1}$ and $\tilde{X}_{2}$ are horizontal lifts of $X_{1}$ and $X_{2}$ respectively.

Note that $R^{\pi}$ is tensorial in $X_{1}, X_{2}$. In other words, a pair of vectors $v_{1}, v_{2} \in$ $T_{q} N$ define a (tangent) vector field $R_{v_{1}, v_{2}}^{\pi}$ on the fiber $\pi^{-1}(\pi(p))$. Namely,

$$
\left(R_{v_{1}, v_{2}}^{\pi}\right)(p):=O_{\tilde{v}_{1}^{p}, \tilde{v}_{2}^{p}}
$$


where $\tilde{v}_{1}^{p}, \tilde{v}_{2}^{p}$ are the unique horizontal vectors of $T_{p} M$ which project down to $v_{1}$ and $v_{2}$ respectively. Observe that $R^{\pi}$ is skew symmetric on $X_{1}, X_{2}$. It may be regarded as a tensor on $N$ with values in an infinite dimensional fiber bundle $\Gamma^{(1,0)}$ over $N$. Namely, $\Gamma_{q}^{(1,0)}$ consists of the tangent field of the fiber $\pi^{-1}(q)$. In the next section we will define, in a natural way, a linear connection in $\Gamma^{(1,0)}$. We will be particularly interested in some parallel finite dimensional vector subbundles of $\Gamma^{(1,0)}$.

Let $M=E$ be a vector bundle over a Riemannian manifold $N$ with a linear connection $\nabla$ (which induces a horizontal distribution $\mathcal{H}$ ). Then the projection to the base is a Riemannian submersion, if $E$ is endowed with the so-called Sasaki metric. Actually, $\left(R_{X_{1}, X_{2}}^{\pi}\right)(p)$ is just the usual curvature tensor $R_{X_{1}, X_{2}}^{\nabla} \cdot p$, if one identifies $T_{p} \pi^{-1}(\{p\})$ with $T_{0} \pi^{-1}(\{p\})=E_{p}$ (see e.g. [1]; this relation will also be clear in the next section).

2.2. Parallel transport and curvature on the fibers. For the sake of simplicity, we will deal here with the global theory, but later (in Subsection 2.4 and Section 3) we will need only the local results which are straightforward to obtain. Assume that $\pi: M \rightarrow N$ is a Riemannian submersion and that $M$ is complete (in order that the horizontal lift of any curve be defined for the whole parameter).

Let $\beta:[0, b] \rightarrow N$ be a (piecewise $\mathcal{C}^{1}$ ) curve and $\psi$ a (tangent vector) field on $\pi^{-1}(\{q\})$, where $q=\beta(0)$. The parallel transport of $\psi$ along $\beta$ is just the field $\left(\tau_{\beta}\right)_{*}(\psi)$ of $\pi^{-1}(\{r\})$, where $r=\beta(b)$. In this way we may regard $\left(\tau_{\beta}\right)_{*}$ as a linear map between fields on $\pi^{-1}(\{q\})$ into fields on $\pi^{-1}(\{r\})$. Now let $X$ be a field on $N$, with associated flow $\phi_{t}$ and let $\tilde{X}$ be the horizontal lift of $X$, with associated flow $\tilde{\phi}_{t}$. Then the horizontal lift through $\tilde{q}$ of the curve $\beta(t)=\phi_{t} . q$ is just $\tilde{\beta}^{\tilde{q}}(t)=\tilde{\phi}_{t} . \tilde{q}$, where $\pi(\tilde{q})=q$. So, the parallel transport of $\psi$ along $\beta$ is just the field $\tilde{\phi}_{t *}(\psi)$.

Now let $\Psi$ be a vertical field on $M$ and $X$ a field on $N$. We define the covariant derivative of $\Psi$ in the direction of $X$ to be

$$
\hat{\nabla}_{X} \Psi=[\tilde{X}, \Psi]=\mathcal{L}_{\tilde{X}}(\Psi)
$$

where $\tilde{X}$ is the horizontal lift of $X$ and $\mathcal{L}$ is the Lie derivative. Observe that the above formula gives rise to a vertical vector since the middle term is $\pi$-related to $[X, 0]=0$. By the well known formula, relating flows and Lie brackets,

$$
\left.\frac{d}{d t}\right|_{0}\left(\phi_{-t}\right)_{*}(\Psi)=[X, \Psi]
$$

one has that

$$
\left(\hat{\nabla}_{X} \Psi\right)_{q}=\left.\frac{d}{d t}\right|_{0}\left(\tau_{\beta_{\mid[0, t]}}\right)_{*}^{-1}\left(\Psi_{\mid \pi^{-1}(\{\beta(t)\})}\right),
$$

with $\beta=\phi_{t} . q$. The vertical field $\Psi$ may be regarded as a section of the infinite dimensional vector bundle $\Gamma^{(1,0)} \rightarrow N$, defined in the previous section, whose elements are vector fields on the fibers (actually, we will not deal with such a big bundle). Following this interpretation, the covariant derivative $\hat{\nabla}_{X} \Psi$ is $\mathcal{C}^{\infty}(N)$ linear on $X$ and $\mathbb{R}$-linear in $\Psi$ (the $\mathbb{R}$-linearity is clear in both variables). Indeed, if $g \in \mathcal{C}^{\infty}(N)$, then the field $g \tilde{X}$, restricted to $\pi^{-1}(\{q\})$, coincides with $g(q) \tilde{X}$. So, $\tilde{g} \tilde{X}$ coincides with $g(q) \tilde{X}$ along the integral curves of $\Psi$ through $\tilde{q}$ (and then one has to calculate the bracket in terms of the flow of $\Psi$ ). 
Moreover, if $f \in \mathcal{C}^{\infty}(N)$, then

$$
\hat{\nabla}_{X} f . \Psi=X(f) . \Psi+f . \hat{\nabla}_{X} \Psi .
$$

Here $f . \Psi$ is the vertical field $\tilde{f} \Psi$ where $\tilde{f}$ is the real function on $M$ which is constant on the fibers and projects down to $f$.

The concept of parallel transport can be defined, like for vector fields, for tensors fields in a given fiber. Now let $\tilde{T}\left(Z_{1}, \ldots, Z_{k}\right)$ be a tensor field on $M$, in the tangent variables $Z_{1}, \ldots, Z_{k}$, with values in the tensor algebra (of a fixed grade $m$ ) of the tangent space of the fibers, if all the variables are vertical. Let $T$ be the restriction of $\tilde{T}$ to vertical fields $Z_{1}, \ldots, Z_{k}$. Such a $T$ will be called a vertical tensor field and it may be regarded as a section on the (infinite dimensional) vector bundle $\Gamma^{(m, k)} \rightarrow N$, where the fiber $\Gamma_{q}^{(m, k)}$ over $q$ consists of the $k$-covariant tensors of $\pi^{-1}(\{q\})$ with values in $\otimes^{m} T \pi^{-1}(\{q\})$.

One can define, as in the case of vertical (tangent) fields, the covariant derivative of $T$ in the direction of a vector field $X$ of $N$. Namely,

$$
\hat{\nabla}_{X} T=\mathcal{L}_{\tilde{X}}(\tilde{T})_{\mid \nu}
$$

where $\mathcal{L}$ denotes the Lie derivative and $\mid \nu$ means that the variables of the tensor have to be restricted to vertical fields. As for vertical vector fields one has a relation, similar to $(*)$, between the covariant derivative and the derivative of the parallel transport.

Given $X, Y$ tangent fields on $N$ one can define the curvature $\hat{R}$ in the obvious way:

$$
\begin{aligned}
\hat{R}_{X . Y} T & =\hat{\nabla}_{X} \hat{\nabla}_{Y} T-\hat{\nabla}_{Y} \hat{\nabla}_{X} T-\hat{\nabla}_{[X, Y]} T \\
& =\mathcal{L}_{\tilde{X}} \mathcal{L}_{\tilde{Y}} \tilde{T}_{\mid \nu}-\mathcal{L}_{\tilde{Y}} \mathcal{L}_{\tilde{X}} \tilde{T}_{\mid \nu}-\mathcal{L}_{[X, Y]^{\sim}} \tilde{T}_{\mid \nu},
\end{aligned}
$$

where $[X, Y]^{\sim}$ is the horizontal lift of the bracket. Observe that one has the well known formula $\mathcal{L}_{\tilde{X}} \mathcal{L}_{\tilde{Y}}-\mathcal{L}_{\tilde{Y}} \mathcal{L}_{\tilde{X}}=\mathcal{L}_{[\tilde{X}, \tilde{Y}]}$. So, since $[X, Y]^{\sim}$ and $[\tilde{X}, \tilde{Y}]$ are both $\pi$-related to $[X, Y], \hat{R}_{X . Y} T=\mathcal{L}_{[\tilde{X}, \tilde{Y}]^{\nu}} T$. Note that $[\tilde{X}, \tilde{Y}]^{\nu}$ is the vertical tensor field $R_{X, Y}^{\pi}$ previously defined (the so-called curvature field). Thus

$$
\hat{R}_{X, Y} T=\mathcal{L}_{R_{X, Y}^{\pi}} T .
$$

In the case of a vertical vector field $\Psi$ the above formula yields

$$
\hat{R}_{X . Y} \Psi=\left[R_{X, Y}^{\pi}, \Psi\right] .
$$

Remark 1. Let $T$ be a vertical tensor field which is parallel, i.e. $\hat{\nabla} T=0$ and let $c$ be any piecewise $\mathcal{C}^{1}$ curve in $N$ from $q$ to $r$. Then the parallel transport along $c$ maps the tensor field $T_{q}$ of $\pi^{-1}(\{q\})$ to the tensor field $T_{r}$ of $\pi^{-1}(\{r\})$ (in particular, the parallel transport of $T_{q}$ is independent of the curve $c$ ).

2.3. Parallel finite dimensional vector subbundles of $\Gamma^{(m, k)}$. In this paper we will not deal with the infinite dimensional vector bundle $\Gamma^{(m, k)} \rightarrow N$ but only with some finite dimensional subbundles $E$ which are parallel. That is, for all $q \in M$, $E_{q}$ is a finite dimensional vector subspace of $\Gamma_{q}^{(m, k)}$. Moreover, if $\beta$ is a curve in $M$ from $q$ to $r$, then the parallel transport $\left(\tau_{\beta}\right)_{*}$ maps (isomorphically) $E_{q}$ into $E_{r}$. In this case it is standard to prove that $E$ is a differentiable vector bundle over $N$ 
(we make this observation since we have not assigned any differentiable structure to $\left.\Gamma^{(m, k)}\right)$. Note that the connection $\hat{\nabla}$ restricts to a linear connection in $E \rightarrow N$.

Remark 2. The linear connection $\hat{\nabla}$ is flat (i.e. $\hat{R}=0$ ) if and only if, for any $T \in E_{q}$, all the Lie derivatives $\mathcal{L}_{R_{X, Y}^{\pi}} T$ are zero, for all $q \in N, X, Y \in T_{q} N$ (see the previous section). For flat connections, the parallel transport is locally independent on the curve chosen from one point to another.

Remark 3. Let $\pi: E \rightarrow N$ be a vector bundle over a Riemannian manifold $N$ with a linear connection $\nabla$. Endow $E$ with the Sasaki metric such that $\pi$ becomes a Riemannian submersion. Then $E$ may be regarded as the finite dimensional subbundle of $\Gamma^{(0,1)}$ which consists of the constant vector fields of the fiber. It is not hard to see that, under this identification, $\nabla=\hat{\nabla}$.

Examples. For our purposes it will be very useful to understand the following two examples which arise from group actions. Let $G$ be a group acting effectively by isometries on a complete Riemannian manifold $M$ such that all orbits are principal (for instance, this is the case if the action is free). Let $N$ be the quotient of $M$ over the $G$-orbits. Then $N$ has a unique Riemannian metric such that the projection $\pi: M \rightarrow N$ is a Riemannian submersion.

(i) Let $E$ be the finite dimensional subbundle of $\Gamma^{(1,0)}$ defined as follows: $E_{q}$ consists of the (intrinsic) Killing fields induced by $G$ on $\pi^{-1}(\{q\})$. Then $E$ is a parallel subbundle of $\Gamma$. Indeed, let $\beta:[0,1] \rightarrow N$ be a curve from $q$ to $r$ and let $\tilde{\beta}^{\tilde{q}}$ be its horizontal lift to $M$ by $\tilde{q}$. Since $G$ preserves the horizontal distribution $\mathcal{H}=\nu^{\perp}$ of $M$, one has that $\tilde{\beta}^{g \cdot \tilde{q}}=g \cdot \tilde{\beta}^{\tilde{q}}$. So,

$$
\tau_{\beta}(g \cdot \tilde{q})=g \cdot \tau_{\beta}(\tilde{q}) .
$$

Now let $X$ belong to the Lie algebra $\mathfrak{g}$ of $G$ and consider the associated Killing field $\bar{X}$ on $M$ defined by $p \mapsto X . p=\left.\frac{d}{d t}\right|_{0} \operatorname{Exp}(t X) . p$. Now replace, in the above formula, $g$ by $\operatorname{Exp}(t X)$ and differentiate it at $t=0$. It yields

$$
\left(\tau_{\beta}\right)_{* \tilde{q}}(X . \tilde{q})=X . \tau_{\beta}(\tilde{q}) .
$$

This means that

Hence, $E$ is parallel.

$$
\left(\tau_{\beta}\right)_{*}\left(\bar{X}_{\mid \pi^{-1}(\{q\})}\right)=\bar{X}_{\mid \pi^{-1}(\{r\})} .
$$

(ii) Let $E$ be the vector subbundle of $\Gamma^{(0,2)}$ whose fiber $E_{q}$ consists of the symmetric $G$-invariant $(0,2)$-tensors on $\pi^{-1}(\{q\})$. Observe that $E_{q}$ is finite dimensional since $G$ acts transitively on $\pi^{-1}(\{q\})$ and therefore a $G$-invariant tensor is completely determined by its value at a given point. In the previous example we have seen that the $G$-action commutes with the parallel transport. This implies that the parallel transport $\left(\tau_{\beta}\right)_{*}$ along a curve $\beta$ in $N$ from $q$ to $r$ maps a $G$-invariant symmetric $(0,2)$-tensor of $\pi^{-1}(\{q\})$ into a $G$-invariant $(0,2)$-tensor of $\pi^{-1}(\{r\})$. Hence, $E$ is parallel. Clearly, the same is true for the subbundle $E$ of $\Gamma^{(m, k)}$ whose fiber $E_{q}$ consists of the $G$-invariant $(m, k)$-tensors on $\pi^{-1}(\{q\})$.

2.4. Homogeneous foliations. In this paragraph, we state a condition which implies that a foliation is locally given by the orbits of the Lie group of the ambient space isometries. This result will be used in the next section to prove our main theorem. Though, for the sake of simplicity, we have worked, up to now, in a global setting, we need to consider local objects (to pass from the global framework to the local one is straightforward). 
For any $q \in N$, let $\mathcal{K}(q)$ be a Lie algebra of Killing vector fields on a fiber $\pi^{-1}(\{q\})$, with the associated pseudogroup of isometries $K(q)$ having the following properties:

(1) For any curve $\beta:[0,1] \rightarrow N$ from $q$ to a nearby point $s$ such that the parallel transport, with respect to the submersion, $\tau_{\beta}(\tilde{q})=\tilde{\beta} \tilde{q}(1)$ is defined, $\left(\tau_{\beta}\right)_{*}$ maps the family $\mathcal{K}(q)$ into the family $\mathcal{K}(s)$.

(2) Any Killing field in the family $\mathcal{K}(q)$ commutes with all the curvature vector fields $R_{X, Y}^{\pi}$.

Theorem 2. Let $\pi: M \rightarrow N$ be a Riemannian submersion. Assume that, on any fiber $\pi^{-1}(\{q\})$, there is a Lie algebra of Killing vector fields $\mathcal{K}(q)$ satisfying properties (1) and (2) above. Then any Killing field in $\mathcal{K}(q)$ extends locally to a vertical Killing field on $M$ (for all $q \in N$ ). In particular, if the pseudogroup of isometries $K(q)$ associated with $\mathcal{K}(q)$ is locally transitive, the vertical foliation is locally homogeneous.

Proof. If $p \in M$, let $F=\pi^{-1}(\{\pi(p)\})$ be a fiber and let $\tilde{\xi}_{1}, \ldots, \tilde{\xi}_{r}$ be basic fields of $M$, which are linearly independent at $p(r$ is the codimension of $F)$. We assume that $F$ is so small such that there exists $\varepsilon>0$ such that $f: F \times(-\varepsilon, \varepsilon)^{r} \rightarrow M$ is a diffeomorphism onto its image, where $f\left(q, s_{1}, \ldots, s_{r}\right)=\tilde{\phi}_{s_{r}}^{r} \ldots \tilde{\phi}_{s_{1}}^{1} . q$ and $\tilde{\phi}^{i}$ is the flow associated to $\tilde{\xi}_{i}$. We may also assume that $M=f\left(F \times(-\varepsilon, \varepsilon)^{r}\right)$ and that the fibers are given by $F_{\left(s_{1}, \ldots, s_{r}\right)}:=f\left(F, s_{1}, \ldots, s_{r}\right)$; this can be done by assuming that the map $\left(s_{1}, \ldots, s_{r}\right) \mapsto \phi_{s_{r}}^{r} \ldots \phi_{s_{1}}^{1} . \pi(q)$ is a global coordinate system of the base $N$, where $\phi^{i}$ is the flow of $\xi_{i}=\pi_{*}\left(\tilde{\xi}_{i}\right)$. Given a Killing field $X \in \mathcal{K}(\pi(p))$, we can define a vertical $\mathcal{C}^{\infty}$ field $Z$ on $M$ which is a Killing field when restricted to any fiber. Namely, the restriction of $Z$ to $F_{\left(s_{1}, \ldots, s_{r}\right)}$ is $\left(\tilde{\phi}_{s_{r}}^{r}\right)_{*} \circ \cdots \circ\left(\tilde{\phi}_{s_{1}}^{1}\right)_{*}(X)=\left(\tau_{\beta}\right)_{*}(X)$, where $\beta$ is the curve in $N$ which starts at $\pi(p)$ and then goes $s_{1}, \ldots, s_{r}$ times along the flows of $\xi_{1}, \ldots, \xi_{r}$ respectively. So, from the assumptions, we have that, for all $q \in N$, the restriction of $Z$ to $\pi^{-1}(\{q\})$ is an element of $\mathcal{K}(q)$. For any such $Z$, the map $\bar{Z}$, defined on $N$ by $q \mapsto Z_{\mid \pi^{-1}(\{q\})}$ will be, by definition, a section of the bundle $\mathcal{K} \rightarrow N$ with fibers $\mathcal{K}(q)$. In this way $\mathcal{K} \rightarrow N$ becomes, in a natural way, a $\mathcal{C}^{\infty}$ vector bundle over $N$. In fact, this bundle is globally trivial. Such a trivialization can be obtained by extending a base of $\mathcal{K}(\pi(p))$ to a base of vector fields on $M$ by means of the above construction. By hypothesis, $\left(\hat{\nabla}_{\xi_{i}} \bar{Z}\right)_{q}=\left[\tilde{\xi}_{i}, Z\right]_{\mid \pi^{-1}(\{q\})}$ is also a section of $\mathcal{K}$. So, $\hat{\nabla}$ is a linear connection in $\mathcal{K}$. From the assumptions such a connection must be globally flat (we assume $N$ to be simply connected) since $\hat{R}_{\xi_{i}, \xi_{j}} \bar{Z}=\left[R_{\xi_{i}, \xi_{j}}^{\pi}, \bar{Z}\right]=0$. This means that the parallel transport $\tau_{\beta}^{\mathcal{K}}$, in the fiber bundle $\mathcal{K} \rightarrow N$, of a Killing field $X \in \mathcal{K}(q)$ does not depend on the curve $\beta$ in $N$ from $q$ to a nearby $q^{\prime}$. It is standard to show that (similarly to the global case) the field $\tau_{\beta}^{\mathcal{K}}(X)$ coincides locally with the field $\left(\tau_{\beta}\right)_{*}(X)$ near $\tau_{\beta}(q)$, if $\tau_{\beta}(q)$ is defined. So, starting with $X \in \mathcal{K}(\pi(p))$, we obtain a parallel section $\hat{X}$ of $\mathcal{K}$, which gives rise to a vertical field of $M$ also denoted by $\hat{X}$. The parallel transport $\tau_{\beta}$, when defined, maps integral curves of $\hat{X}$ into integral curves of $\hat{X}$. Now let $\beta:[0, \varepsilon]$ be a short curve in $N$ starting at $\pi(u)$ (in order that the horizontal lift $\tilde{\beta}^{u}$ be defined). Let $\tau_{s}$ be the parallel transport along the curve $\beta_{\mid[0, s]}$ and $\varphi_{t}$ be the local flow of $\hat{X}$. We want to prove, for any fixed $t$, that $\left(\varphi_{t}\right)_{* p}$ is a linear isometry and hence $\hat{X}$ will be a Killing field on $M$. Since $\hat{X}$ is a Killing field on the fiber $\pi^{-1}(\{\pi(u)\})$ we need only to prove that $\left(\varphi_{t}\right)_{* p}$ preserves the norm of vectors in the horizontal 
space $\mathcal{H}_{p}$. One has that

or equivalently,

$$
\varphi_{t} . \tau_{s}(p)=\tau_{s}\left(\varphi_{t} . p\right)
$$

$$
\varphi_{t} \cdot \tilde{\beta}^{p}(s)=\tilde{\beta}^{\varphi_{t} \cdot p}(s) .
$$

Differentiating the above expression with respect to $s$ (at $s=0$ ) yields

$$
\left(\varphi_{t}\right)_{* p}\left(\left(\tilde{\beta}^{p}\right)^{\prime}(0)\right)=\left(\tilde{\beta}^{\varphi_{t} \cdot p}\right)^{\prime}(0) ;
$$

but the horizontal vectors $\left(\tilde{\beta}^{p}\right)^{\prime}(0)$ and $\left(\tilde{\beta}^{\left(\varphi_{t} \cdot p\right)}\right)^{\prime}(0)$ have the same norm, since they both project down to $\beta^{\prime}(0)$. Since $\beta$ is arbitrary, we conclude that $\left(\varphi_{t}\right)_{* p}$ is a linear isometry.

Example 2. Let $N^{n}$ be a simply connected rank one symmetric space, with connected isometry group $G$, and let $\pi: S(N) \rightarrow N$ be the unit tangent bundle. Let $\mathcal{K}(q)$ be the set of Killing fields on the unit sphere $S(N)_{q}$ of $T_{q} N$, induced by the isotropy $G_{q}$ (which coincides with the holonomy group at $q$ ). The parallel transport in $N$ maps, isometrically, fibers into fibers. Moreover, we have that the family $\{\mathcal{K}(q)\}_{q \in N}$ is invariant under parallel transport. Observe that any fiber $S(N)_{q}$ is extrinsically homogeneous in $S(N)$, since $G_{q}$ acts transitively on the sphere $S(N)_{q}$; but the vertical foliation is not a homogeneous foliation. In fact, assume that $S(N)_{q}=H . q$, for some group of isometries $H$ of $S(N)$, for any $q \in N$. Choose an element $\tau_{\beta}$ of the holonomy group of $N$ at $q$, where $\beta$ is a loop based at $q$. Note that $H$ must preserve the horizontal distribution of $S(N)$ since it preserves the vertical distribution. So, an element of $H$ maps horizontal curves into horizontal curves (starting at the same fiber). Thus, any element $h \in H$, restricted to $S(N)_{q}$, commutes with $\tau_{\beta}$. Assume now that $\tau_{\beta}(v)=v$, for some $v \in S(N)_{q}$. So, $h . v=h . \tau_{\beta}(v)=\tau_{\beta}(h . v)$. Thus, $\tau_{\beta}$ is the identity transformation of $S(N)_{q}$, since $H$ is transitive on the fibers. Then the holonomy group of $N$ at $\pi(v)$ has no isotropy at $v$. This is a contradiction for any symmetric space.

2.5. Invariant Weyl tensors. A tensorial Weyl invariant is a tensor $T$ that one can naturally define in any Riemannian manifold $M^{n}$ in terms of the metric tensor $\langle$,$\rangle , the curvature tensor R$ and all of its covariant derivatives $\nabla^{k} R$. Moreover, any coefficient $T_{J}^{I}\left(I=\left(i_{1}, \ldots, i_{l}\right), J=\left(j_{1}, \ldots, j_{s}\right)\right)$ of this tensor, with respect to any orthonormal basis $e_{1}, \ldots, e_{n}$, is a (fixed) polynomial in the components of the curvatures tensor and all of its derivatives up to a fixed order. In particular, the (formal) expression of $T_{J}^{I}$ does not depend on the chosen orthonormal basis. If $T$ is a function, i.e., a real-valued tensor of type $(0,0)$, then $T$ is called a scalar Weyl invariant.

In our article we will not consider real-valued mixed tensors of type $(k, m)$. Instead of that we will always use tensors of type $(0, m)$ with values in the $k$ tensorial algebra of the tangent space. Of course, this point of view is equivalent, but it will be more suitable for our purposes. This is done in order to extend, in a canonical and simple way, families of tensors on the leaves of a given foliation to a tensor of the ambient space.

\section{Examples of tensorial Weyl invariants.}

- $R_{X, Y}$, tensor of type $(0,2)$ with values in the skew-symmetric endomorphisms of the tangent space.

- $R_{X, Y} Z$, tensor of type $(0,3)$ with values in the tangent space (of course, it may be regarded as a $(1,3)$ tensor with real values). 
- $\left\langle R_{X . Y} Z, W\right\rangle$, tensor of type $(0,4)$ (with real values).

- $\|R\|^{2}=\sum_{i, j, k, l}\left\langle R_{e_{i} . e_{j}} e_{k}, e_{l}\right\rangle^{2}$, tensor of type $(0,0)$ (i.e. a scalar Weyl invariant)

- The Ricci form $\operatorname{ric}(X, Y)=\left\langle\sum_{i} R\left(X, e_{i}\right) e_{i}, Y\right\rangle$, symmetric tensor of type $(0,2)$.

- The Ricci tensor $\operatorname{Ric}(X)=\sum_{i} R\left(X, e_{i}\right) e_{i}$, tensor of type $(0,1)$ with values in the tangent space.

- $\left\langle\left(\nabla_{R_{X, Y} Z} R\right)_{R i c(U), V} W, H\right\rangle$, tensor of type $(0,7)$.

- The sum and the tensor product of any two tensorial Weyl invariants.

- The tensor of type $(k+1, m-1)$ (resp. of type $(k-1, m+1))$ obtained by transforming a covariant (resp. contravariant) variable into a contravariant (resp. covariant) variable in a tensorial Weyl invariant.

- The tensor obtained by contracting any two variables of a tensorial Weyl invariant.

- The tensor obtained by replacing, one variable of a tensorial Weyl invariant (let us say covariant), by a vectorial Weyl invariant.

- The covariant derivative $\nabla T$ of a tensorial Weyl invariant $T$.

- The gradient of a scalar Weyl invariant.

- The Lie derivative $\mathcal{L}_{X} T$ of a tensorial Weyl invariant $T$ in the direction of a vectorial Weyl invariant $X$. In particular, $[X, Y]$ is a vectorial Weyl invariant if $Y$ is also a vectorial Weyl invariant.

- The square of the norm a tensorial Weyl invariant (gives rise to a scalar Weyl invariant).

- Let $p$ be a polynomial defined on the vector space $V$ of algebraic tensors of some type in $\mathbb{R}^{n}$, which is invariant under the full orthogonal group $O(n)$ (acting on $V$ in the obvious way). Let $T$ be a tensorial Weyl invariant of the same type. Then $p(T)$ is a scalar Weyl invariant. To calculate $p(T)$, one has to identify tangent spaces with the Euclidean space by means of a linear isometry (and this does not depend on the linear isometry chosen because of the $O(n)$-invariance of $p$ ).

It follows from the Weyl theory of invariants that the scalar Weyl invariants are obtained as a linear combination of complete traces (with respect to some paring of the indices) of tensors of the form

$$
\left\langle\nabla^{m_{1}} R,\right\rangle \ldots\left\langle\nabla^{m_{s}} R,\right\rangle
$$

where $m_{1}, \ldots, m_{s}$ are greater or equal to $0\left(\nabla^{0} R:=R\right)$.

\section{LEVEL SETS OF SCALAR WEYL INVARIANTS}

Let $M^{n}$ be a Riemannian manifold and let $p \in M$ be such that the gradients of all the scalar Weyl invariants span a maximal dimensional subspace, let us say $\nu_{p} \subset$ $T_{p} M$. Let $f_{1}, \ldots, f_{k}$ be scalar Weyl invariants such that $\operatorname{grad}\left(f_{1}\right)(p), \ldots, \operatorname{grad}\left(f_{k}\right)(p)$ is a basis of $\nu_{p}$. In some neighborhood $U$ of $p$ in $M$, the vectorial Weyl invariants $\operatorname{grad}\left(f_{1}\right), \ldots, \operatorname{grad}\left(f_{k}\right)$ are linearly independent and hence the regular level sets of $f_{1}, \ldots, f_{k}$ in $U$ coincide with the level sets of the family of scalar Weyl invariants. Observe that any level set is a submanifold of codimension $k$ in $U$. Since we will work locally, we may assume, without loss of generality, that $U=M$. Now let $S_{1}, \ldots, S_{s}$ be tensorial Weyl invariants and consider $S=S_{1} \otimes \cdots \otimes S_{s}$. Identify, for any $x \in M, T_{x} M$ with $\mathbb{R}^{n}$ by means of a linear isometry $h_{x}$. Let $\mathbb{W}$ be the tensor algebra of $\mathbb{R}^{n}$ of the same type as $S$ (so that $h_{x}\left(S_{x}\right) \in \mathbb{W}$ ). Let $p_{1}, \ldots, p_{r}$ be polynomials in $\mathbb{W}$ that distinguish the orbits of the orthogonal group $O(n)$ in 
$\mathbb{W}$. Then $g_{i}(x):=p_{i}\left(h_{x}\left(S_{x}\right)\right), i=1, \ldots, r$, does not depend on the chosen isometries $h_{x}$, because of the $O(n)$-invariance, and so it defines a scalar Weyl invariant (see [6]). If $F$ is a level set of $f_{1}, \ldots, f_{k}$, then $g_{1}(x), \ldots, g_{r}(x)$ are constant on $F$. This implies that for any $x \in M$ there exists a linear isometry $d_{x}: T_{p} M \rightarrow T_{x} M$ which maps $S_{p}$ into $S_{x}$. In particular, $d_{x}\left(\left(S_{1}\right)_{p}\right)=\left(S_{1}\right)_{x}, \ldots, d_{x}\left(\left(S_{r}\right)_{p}\right)=\left(S_{r}\right)_{x}$. Then it is not hard to see that, for any $x \in M$, there exists a linear isometry $\bar{d}_{x}: T_{p} M \rightarrow T_{x} M$ such that, for any tensorial Weyl invariant $S, \bar{d}_{x}\left(S_{p}\right)=S_{x}$. Observe that $\bar{d}_{x}\left(\operatorname{grad}\left(f_{1}\right)(p)\right)=\operatorname{grad}\left(f_{1}\right)(x)$, for all $i=1, \ldots, k$ (in particular, $\left.\bar{d}_{x}\left(T_{p} F\right)=T_{x} F\right)$. Thus the functions $\left\langle\operatorname{grad}\left(f_{i}\right), \operatorname{grad}\left(f_{j}\right)\right\rangle$ are constant on any level set (this could have been observed from the fact that $\left\langle\operatorname{grad}\left(f_{i}\right), \operatorname{grad}\left(f_{j}\right)\right\rangle$ are scalar Weyl invariants). From this fact it is not difficult to show that there exists a Riemannian metric on the quotient manifold $N$, obtained by identifying points in the same level set, such that the projection $\pi: M \rightarrow N$ is a Riemannian submersion (possibly by making $M$ smaller). The horizontal fields with respect to this submersion $\xi_{1}=\operatorname{grad}\left(f_{1}\right), \ldots, \xi_{k}=\operatorname{grad}\left(f_{k}\right)$ are basic.

Now let $F$ be a fixed level set. By making (constant) linear combination of the scalar Weyl invariants $f_{1}, \ldots, f_{k}$ we may assume that $\xi_{1}=\operatorname{grad}\left(f_{1}\right), \ldots, \xi_{k}=$ $\operatorname{grad}\left(f_{k}\right)$ is an orthonormal frame of the normal space along $F$ (this will not be in general true for other level sets).

Let us consider the following tensorial Weyl invariant on $M$ of type $(1,1)$ :

$$
\operatorname{pr}(v)=v-\sum_{i=1}^{k}\left\langle v, \xi_{i}\right\rangle \xi_{i} .
$$

Then $\operatorname{pr}(v)$ coincides, along $F$, with the orthogonal projection to the tangent space.

Remark 4. If $X$ is a vectorial Weyl invariant, then its projection to $F$ coincides with the restriction to $F$ of the vectorial Weyl invariant $\operatorname{pr}(X)$.

Lemma 1. Let $T$ be a tensorial Weyl invariant on $M$ of type $(0, m)$ and let $\bar{T}$ be its restriction to the tangent space $T F$ of the level set $F$. Let $\bar{\nabla}$ be the Levi-Civita connection of $F$. Then $\bar{\nabla} \bar{T}$ is the restriction to $T F$ of a tensorial Weyl invariant of $M$ of type $(0, m+1)$.

Proof. Let us consider the $(0, k)$-tensorial Weyl invariant

$$
\tilde{T}\left(v_{1}, \ldots, v_{m}\right)=T\left(\operatorname{pr}\left(v_{1}\right), \ldots, \operatorname{pr}\left(v_{m}\right)\right) .
$$

It is easy to check that the restriction to $T F$ of the $(0, m+1)$-tensorial Weyl invariant $\nabla \tilde{T}$ coincides with $\bar{\nabla} \bar{T}$, where $\nabla$ is the Levi-Civita connection of $M$.

Lemma 2. (i) The second fundamental form $\alpha$ of $F$ is the restriction to TF of a tensorial Weyl invariant of $M$.

(ii) The curvature tensor $\left\langle\bar{R}_{X, Y} Z, W\right\rangle$ of $F$ is the restriction to TF of a tensorial Weyl invariant.

Proof. (i) Let us consider the tensorial Weyl invariant $T_{i}(X, Y)=-\left\langle\nabla_{X} \xi_{i}, Y\right\rangle \xi_{i}=$ $\left\langle\alpha(X, Y), \xi_{i}\right\rangle \xi_{i}$. Then $\alpha(X, Y)=\bar{T}_{1}(X, Y)+\cdots+\bar{T}_{k}(X, Y)$, where $\bar{T}_{i}$ is the restriction to $T F$ of $T_{i}$.

(ii) Let $\left\langle R_{X, Y} Z, W\right\rangle$ be the curvature tensor of the ambient space. Then, if $X, Y, Z, W$ are tangent to $F$, we have the well known Gauss formula

$$
\left\langle\bar{R}_{X, Y} Z, W\right\rangle=\left\langle R_{X, Y} Z, W\right\rangle+\langle\alpha(Y, Z), \alpha(X, W)\rangle-\langle\alpha(X, Z), \alpha(Y, W)\rangle,
$$


where $R$ is the curvature tensor of the ambient space. Applying part (i) we obtain the second part.

Corollary 1. The level set $F$ is locally homogeneous. Moreover, there exists a locally transitive pseudo Lie group $G$ of isometries of $F$ with the following property: if $\bar{T}$ is a tensor on $F$ which coincides with the restriction to TF of a tensorial Weyl invariant, then $\bar{T}$ is $G$-invariant.

Proof. Given $p_{1}, p_{2} \in F$ there exists a linear isometry $\mathcal{I}: T_{p_{1}} M \rightarrow T_{p_{2}} M$ which maps any tensorial Weyl invariant at $p_{1}$ into the same invariant at $p_{2}$. Moreover, $\mathcal{I}\left(T_{p_{1}}\right)=T_{p_{2}}$. From the previous two lemmas one obtains that $\mathcal{I}$ maps $\left(\overline{\nabla^{j}} \bar{R}\right)_{p_{1}}$ into $\left(\bar{\nabla}^{j} \bar{R}\right)_{p_{2}}$, for all $j \geq 0$. Then by the theorem of Singer [7], we mentioned in the Introduction, $M$ is locally homogeneous (a new proof of this fact was given in [3]). It is standard that one can define a differentiable subbundle $B$ of the frame bundle of $F$ such that the linear isometry which relates any pair of basis in $B$ preserves all the tensorial Weyl invariants. Then, by imitating the construction of the canonical connection $\nabla^{c}$ in [3] in this suitable subbundle, we obtain the second assertion (with respect to this connection all tensorial Weyl invariants are parallel). So, any tensorial Weyl invariant must be invariant under the (pseudo) Lie group of transvections $G$ of $\nabla^{c}$ (which is locally transitive).

Remark 5. (i) The projection to $T F$ of any vectorial Weyl invariant is invariant under the pseudo Lie group $G$. This is because the projection $p r$ to $T F$ is the restriction of a tensorial Weyl invariant. In particular, all curvature vector fields $R_{X, Y}^{\pi}, X, Y \in T_{\pi(p)} N, p \in F$ are $G$-invariant. Indeed, $R_{X, Y}^{\pi}=[\tilde{X}, \tilde{Y}]^{\nu}$ (recall that $\left.\nu_{\mid F}=T F\right)$ and the horizontal lifts of $\tilde{X}, \tilde{Y}$, along $F$, must be linear combinations of the basic fields $\xi_{1}, \ldots, \xi_{k}$. The assertion follows from the fact that $\left[\xi_{i}, \xi_{j}\right]_{\mid F}^{\nu}$ coincides with the restriction to $F$ of a vectorial Weyl invariant. Note that a field $Z$ of $F$ is $G$ invariant if and only if $[H, Z]=0$ for any Killing field $H$ induced by by $G$.

(ii) Observe that a tensorial Weyl invariant which vanishes at some point of $F$ must be identically zero along $F$, since it is algebraically constant along $F$.

We will now construct two finite dimensional subspaces of tensors on $F$. The first, denoted by $E_{\pi(p)}^{(0,2)}$, consists of $(0,2)$ tensors and includes the metric tensor $(p \in F)$. The second subspace, denoted by $E_{\pi(p)}^{(1,0)}$ consists of vector fields, i.e., $(1,0)$ tensors, and includes all the curvature vectors fields $R_{X, Y}^{\pi}$ of $F$. All the elements of both subspaces are the restriction to $F$ of tensorial Weyl invariants.

(i) $E_{\pi(p)}^{(0,2)}$ consists of the linear span of the restriction to $F$ of the iterated Lie derivatives of the metric tensor $\left(\mathcal{L}_{\xi_{i_{1}}} \ldots \mathcal{L}_{\xi_{i_{r}}}\langle,\rangle\right)_{\mid F}$.

(ii) $E_{\pi(p)}^{(1,0)}$ consists of the linear span of the projection to $F$ of the iterated Lie brackets $\left[\xi_{i_{1}},\left[\xi_{i_{2}},\left[\ldots,\left[\xi_{i_{r-1}}, \xi_{i_{r}}\right]\right]^{\nu}\right.\right.$.

Both subspaces are finite dimensional due to part (ii) of the previous remark.

Of course we can do the same construction for any level set (i.e., any fiber of the submersion $\pi: M \rightarrow N)$.

So, we get vector bundles $E^{(0,2)}$ and $E^{(1,0)}$ over $N$. We may assume, by considering a nearby base point and making, possibly, $M$ smaller, that the dimensions of the subspaces are constant on $N$. It is standard to prove that this yields differentiable (finite dimensional) vector bundles $E^{(0,2)} \rightarrow N$ and $E^{(1,0)} \rightarrow N$ (in fact, a 
rigorous proof of this can be done by following the ideas in the proof of Theorem 2 , combined with the next lemma).

Lemma 3. The vector bundles $E^{(0,2)} \rightarrow N$ and $E^{(1,0)} \rightarrow N$ are parallel subbundles of $\Gamma^{(0,2)}$ and $\Gamma^{(1,0)}$ respectively, with respect to the connection $\hat{\nabla}$.

Proof. The fact that $E^{(0,2)}$ is parallel follows from the construction. For the second bundle, this is not immediate since we project down to the tangent to the fibers only at the last step and the vector fields we obtain are the restriction of vectorial Weyl invariants. Observe that $\left[\xi_{i}, \xi_{j}\right]^{\nu}$ restricted to any fiber is the restriction of a vectorial Weyl invariant, but this invariant may change from fiber to fiber.

Let $X=\left[\xi_{i_{1}},\left[\xi_{i_{2}},\left[\ldots,\left[\xi_{i_{r-1}}, \xi_{i_{r}}\right]\right]\right.\right.$ and let $\bar{\xi}_{i}$ be the projection to $N$ of the basic vector field $\xi_{i}$. The field $X$ is projectable (but not in general basic). Let us denote its projection to $N$ by $\bar{X}$. Observe that $X-X^{\nu}$ is basic and projects down to $\bar{X}$ (and so, along $F$, it is a linear combination of $\xi_{1}, \ldots, \xi_{k}$ ).

We have that $\hat{\nabla}_{\bar{\xi}_{i}} X^{\nu}$ at a point $q \in N$ is the field $\left[\xi_{i}, X^{\nu}\right]$ of the level set $\pi^{-1}(\{q\})$ (due to the fact that $\left[\xi_{i}, X^{\nu}\right]$ is vertical, since it is $\pi$-related to $\left[\bar{\xi}_{i}, 0\right]=0$ ). One has that

$$
\begin{aligned}
{\left[\xi_{i}, X^{\nu}\right] } & =\left[\xi_{i}, X^{\nu}\right]^{\nu}=\left[\xi_{i}, X-\left(X-X^{\nu}\right)\right]^{\nu}=\left[\xi_{i}, X\right]^{\nu}-\left[\xi_{i},\left(X-X^{\nu}\right)\right]^{\nu} \\
& =\left[\xi_{i}, X\right]^{\nu}-R_{\bar{\xi}_{i}, \bar{X}}^{\pi} \in E_{\pi(p)}^{(1,0)} .
\end{aligned}
$$

Proof of Theorem 1. We keep the notation and assumptions of this section. Define, for any $q \in N, \mathcal{K}(q)$ to be set of the (locally defined) Killing fields on the fiber $\pi^{-1}(\{q\})$ such that its associated flows preserve any of the vector fields of $E_{q}^{(1,0)}$ and any of the elements of $E_{q}^{(0,2)}$ (which are symmetric tensors of type $(0,2)$ ). We have that $\mathcal{K}(q)$ is a Lie algebra and its associated Lie pseudogroup $G(q)$ is (locally) transitive on the fiber, due to Corollary 1 . Let $\beta:[0,1] \rightarrow N$ be a curve from $q$ to a nearby $s$. Let $H \in \mathcal{K}(q)$ and let us consider the field $Z=\left(\tau_{\beta}\right)_{*}(H)$ of $\pi^{-1}(\{s\})$. One has that the flow of $Z$ must preserve any element of $\left(\tau_{\beta}\right)_{*}\left(E_{q}^{(1,0)}\right)=E_{q}^{(1,0)}$ and any element of $\left(\tau_{\beta}\right)_{*}\left(E_{q}^{(0,2)}\right)=E_{q}^{(0,2)}$. In particular, $Z$ is a Killing field since the metric tensor of $\pi^{-1}(\{s\})$ belongs to $E_{q}^{(0,2)}$. From the maximality of $\mathcal{K}(s)$ we obtain that $Z \in \mathcal{K}(s)$. Thus, the family $\{\mathcal{K}(q)\}_{q \in N}$ is invariant under parallel transport. Moreover, if $H \in \mathcal{K}(q)$, then $H$ commutes with all the curvature vector fields (since its associated flow leaves invariant any of the elements of $E_{q}^{(1,0)}$ ). Then, by Theorem 2, we conclude that the foliation by the level sets of the scalar Weyl invariants is locally homogeneous. This shows that the (local) cohomogeneity of $M$ coincides with the codimension of the foliation by regular level sets of the scalar Weyl invariants.

Remark 6. Let $p \in M$ and let $D_{p}$ be the linear span of $\left\{X(p): X \in E_{\pi(p)}^{(1,0)}\right\}$. Then $\mathcal{H}_{p} \oplus D_{p}$ is the tangent space to the so-called holonomy subbundle by $p$ (that is, the points that can be locally reached by horizontal curves starting at $p$; cf. [8]).

Remark 7. Observe that, locally, $p$ and $q$ belong to the same regular level set of the scalar Weyl invariants if and only if there exists a linear isometry from $T_{p} M$ into $T_{q} M$ which maps all the covariant derivatives of the curvature tensor $\left(\nabla^{j} R\right)_{p}$ into $\left(\nabla^{j} R\right)_{q}$. This follows from the Weyl theory of invariants. 


\section{REFERENCES}

1. P. Dombrowski, On the geometry of the tangent bundle, J. Reine Angew. Math. 210 (1962), 73-88. MR0141050 (25:4463)

2. D. B. A. Epstein, Natural tensors on Riemannian manifolds, J. Differ. Geom. 10 (1975), 631-645. MR0415531 (54:3617)

3. L. Nicolodi, F. Tricerri, On two theorems of I. M. Singer about homogeneous spaces, Ann. Global Anal. Geom. 8 (1990), no. 2, 193-209. MR1088511 (92b:53075)

4. B. O'Neill, The fundamental equation of a submersion, Michigan Math. J. 13 (1966), 459-469. MR0200865 (34:751)

5. F. Podestà, A. Spiro, Cohomogeneity one manifolds and hypersurfaces of the Euclidean space, Ann. Global Anal. Geom. 13 (1995), 169-184. MR1336212 (96e:53010)

6. F. Prüfer, F. Tricerri and L.Vanhecke, Curvature invariants, differential operators and locally homogeneity, Transactions Amer. Math. Soc. 348 No. 11 (1996), 4643-4652. MR1363946 (97a:53074)

7. I.M. Singer, Infinitesimally homogeneous spaces, Comm. Pure Appl. Math. 13 (1960), 685-697. MR0131248 (24:A1100)

8. H. J. Sussmann, Orbits of families of vector fields and integrability of distributions, Transactions Amer. Math. Soc. 180 (1973), 171-188. MR0321133 (47:9666)

Dipartimento di Matematica, Università di Torino, via Carlo Alberto 10, 10123 TORINO, ITALY

E-mail address: sergio.console@unito.it

Famaf, Universidad Nacional de Córdoba, Ciudad Universitaria, 5000 Córdoba, ArGENTINA

E-mail address: olmos@mate.uncor.edu 